\title{
Nanoengineering Neural Stem Cells on Biomimetic Substrates Using Magnetofection Technology
}

Christopher F. Adams, ${ }^{1 *}$ Andrew W. Dickson, ${ }^{2}$ Jan-Herman Kuiper, ${ }^{3}$ Divya M. Chari ${ }^{1,2}$

1. Institute of Science and Technology in Medicine, Keele University, Newcastle-under-Lyme, ST5 $5 B G, U K$

2. School of Medicine, Keele University, Newcastle-under-Lyme, ST5 5BG, UK

3. Institute of Science and Technology in Medicine, The Robert Jones and Agnes Hunt Orthopaedic Hospital, Oswestry, SY10 7AG, UK

*corresponding author: c.adams@keele.ac.uk

\section{Abstract}

Tissue engineering studies are witnessing a major paradigm shift to cell culture on biomimetic materials that replicate native tissue features from which the cells are derived. Few studies have been performed in this regard for neural cells, particularly in nanomedicine. For example, platforms such as magnetic nanoparticles (MNPs) have proven efficient as multifunctional tools for cell tracking and genetic engineering of neural transplant populations. However, as far as we are aware, all current studies have been conducted using neural cells propagated on non-neuromimetic substrates that fail to represent the mechano-elastic properties of brain and spinal cord microenvironments. Accordingly, it can be predicted that such data is of less translational and physiological relevance than that derived from cells grown in neuromimetic environments. Therefore, we have performed the first test of magnetofection technology (enhancing MNP delivery using applied magnetic fields with significant potential for therapeutic application) and its utility in genetically engineering neural stem cells (NSCs; a population of high clinical relevance) propagated 
in biomimetic hydrogels. We demonstrate magnetic field application safely enhances MNP mediated transfection of NSCs grown as 3D spheroid structures in collagen which more closely replicates the intrinsic mechanical and structural properties of neural tissue than routinely used hard substrates. Further, as it is well known that MNP uptake is mediated by endocytosis we also investigated NSC membrane activity grown on both soft and hard substrates. Using high resolution scanning electron microscopy we were able to prove that NSCs display lower levels of membrane activity on soft substrates compared to hard, a finding which could have particular impact on MNP mediated engineering strategies of cells propagated in physiologically relevant systems.

Keywords: Magnetic nanoparticle, transfection, neural stem cell, biomimetic hydrogel, electron microscopy, membrane activity

\section{Introduction}

Neural stem cells (NSCs) can promote endogenous/transplant mediated regeneration and functional recovery in a range of animal models of neurological injury and disease. ${ }^{1}$ Several clinical trials are currently underway to test the therapeutic efficacy of these cells, including in Alzheimer's disease, spinal cord injury (SCl) and lysosomal storage disorders. ${ }^{2}$ Further, in recent years, magnetic nanoparticles (MNPs) have been proven to be an important nanomedicine platform to augment the neuro-regenerative properties of NSCs, by mediating gene delivery and non-invasive cell tracking (by acting as contrast agents for magnetic resonance imaging). ${ }^{3-6}$ Investigating MNP handling, functional utility and toxicity in NSCs is therefore of critical importance to developing optimised particle designs and protocols for therapeutic application. Several studies have reported safe and efficient engineering of NSCs using MNPs for both labelling and gene delivery approaches ${ }^{5,7,8}$ with detailed characterisation of NSC membrane responses to these biomaterials. ${ }^{9}$ For example, it was shown that increased levels of NSC membrane activity were predictive of enhanced MNP uptake (specifically when MNP physicochemical properties such as magnetite content were altered) suggesting this is a 
key cellular feature for predicting NSC handling of nanoparticles. ${ }^{9}$ Whilst useful, it is crucial to note that these studies and the associated biological readouts (including particle uptake, membrane properties, transfection efficiency and safety) reported so far in the nanomedicine literature have, as far as we are aware, all been conducted using NSCs propagated in 2D cultures on hard substrates such as glass coverslips or plastic culture dishes.

However, it should be underlined that cells within the body reside in 3D tissue structures with different elastic moduli, with growing evidence that this specific property has a profound effect on cell behaviour and function. As such, there is a major current drive to culture NSCs using 'soft' substrates which more closely mimic the intrinsic structural and physical properties of neural tissue in vivo. This is both for basic biological research (e.g. to recreate NSC niches) ${ }^{10}$ and for further clinical application (e.g. as part of implantable and supportive cellular matrices) ${ }^{11}$ Indeed, NSC biological properties including migration, proliferation and differentiation are altered by varying substrate stiffness ${ }^{12-15}$ suggesting this feature has a significant measure of control over NSC biology. In terms of nanoparticle handling, it has been shown that bovine aortic endothelial cells increase particle uptake on softer substrates - a finding the authors attribute to 'less tense' membranes able to participate in more efficient endocytosis. ${ }^{16}$ It has also been reported that the efficiency of non-viral gene delivery to murine MC3T3-E1 preosteoblasts can be enhanced by increasing substrate stiffness, as a result of both increased cellular uptake of the transfection complexes and accelerated dissociation of DNA from the complex to facilitate its transcription. ${ }^{17}$ This suggests that in substrate responsive cells (such as NSCs), the cellular environment plays a crucial role in determining cell handling and functional utility of MNPs - with key implications for therapeutic MNP application. Despite this, to the best of our knowledge, no study has ever evaluated the efficacy of MNP mediated engineering of NSCs grown on biomimetic substrates, although the latter is likely to offer more biologically relevant insights into MNP handling by NSCs, compared with cells grown on artificial and 'hard' 2-D surfaces. 
Further, it is well known that nanoparticle uptake/engineering of cells depends critically on membrane activity and is mediated by a range of endocytotic mechanisms. However, it has never been established if stem cell membrane features differ when grown on hard versus soft substrates- a question of significant importance when considering strategies to engineer these cells using MNPs. In this context, we recently described a high resolution field emission scanning electron microscopy based method (termed OTOTO-FESEM) wherein various parameters of stem cell membrane activity (which correlate with nanoparticle uptake) can be examined and quantified at high resolution. ${ }^{9}$ The feasibility of applying this methodology to study NSC membrane activity in soft matrices has never been established.

Therefore, the specific objectives of this study are to (i) investigate the feasibility of genetically engineering intraconstruct NSCs propagated as 3D spheroids in collagen hydrogels (which mimic the fibrous nature of the extracellular matrix [ECM] and soft mechanical properties of the CNS) using magnetofection procedures (MNPs plus magnetic field application) and (ii) assess whether the OTOTO-FESEM technique can be used to examine/quantify membrane features of NSCs in such soft materials, to provide an explanation for the observed differences between cells grown in materials of differing stiffness.

\section{Materials and methods}

\subsection{Reagents}

Cell culture reagents were from Invitrogen (Paisley, Scotland, UK) and Sigma (Poole, Dorset, UK). Basic fibroblast growth factor (FGF-2) was from Peprotech (Rocky Hill, NJ, USA) and epidermal growth factor (EGF) was from R\&D systems Ltd (Abingdon, UK). Penicillin and streptomycin were from Fisher (Loughborough, UK). Accutase was from Sigma and DNase I was from Roche (Welwyn, UK). Cell culture grade plastics were purchased from Fisher. Type I, rat-tail collagen was from Corning (Tewkesbury, MA, USA) and powdered Gibco MEM $\alpha$ was purchased from Life Technologies. 
The LIVE/DEAD Viability/Cytotoxicity Assay Kit was from Invitrogen (Paisley, UK). Neuromag MNPs were purchased from OzBiosciences (Marseilles, France) and are reported to have a hydrodynamic diameter of ca. $160 \mathrm{~nm} .{ }^{18}$ Culture plates were placed on a 24 magnet array (magnefect nano, Nanotherics, Stoke-on-Trent, UK) for magnetic field experiments. Oscillation frequency is controlled by an external computer and amplitude for all experiments was $0.2 \mathrm{~mm}$. Primary antibodies were rabbit anti- $\beta$-tubulin (Tuj-1) from Covance (Princeton, NJ), rat anti-myelin basic protein (MBP) from Serotec (Kidlington, UK), rabbit anti-glial fibrillary acidic protein (GFAP) from DakoCytomation (Ely, UK), mouse anti-nestin from BD Biosciences (Oxford, UK) and rabbit anti-Sox-2 from Millipore (Watford, UK). Secondary antibodies were from Jackson Immunoresearch Laboratories Ltd (Westgrove, PA, USA). Vectashield mounting medium with 4,6-diamidino-2-phenylindole (DAPI) was from Vector Laboratories (Peterborough, UK). The pmaxGFP plasmid (size $3.5 \mathrm{~kb}$; encodes green fluorescent protein [GFP]) was from Amaxa Biosciences (Cologne, Germany). The care and use of all animals used for cell culture were in accordance with the Animals (Scientific Procedures) Act of 1986 (UK) with approval by the local ethics committee.

\subsection{NSC derivation and maintenance}

NSCs were derived by removal and dissociation (by DNAse I) of the subventricular zone (SVZ) from CD1 mice (post-natal days 1-3). Cells were maintained in neurosphere medium (3:1 mix DMEM:F12, 2\% B27, $25 \mathrm{ng} / \mathrm{ml} \mathrm{EGF}$ and FGF, $5 \mathrm{ng} / \mathrm{ml}$ heparin, $50 \mathrm{U} / \mathrm{ml}$ penicillin and $50 \mu \mathrm{g} / \mathrm{ml}$ streptomycin) at $37^{\circ} \mathrm{C}$ and $5 \% \mathrm{CO}_{2}$. Under growth factor drive NSCs are preferentially selected and form balls of floating cells termed neurospheres. NSCs were routinely passaged using a mixture of Accutase/DNAse I and cells from passages 1-3 were used for all experiments.

\subsection{Collagen hydrogel formation}

In this study NSCs were added to pre-formed collagen hydrogels in 24 well plates where they form 3D aggregates of cells, termed spheroids. For gel formulation collagen was dissolved in acetic acid to the required concentration before addition of $10 X \mathrm{MEM} \alpha$. This solution was neutralised with $\mathrm{NaOH}$ 
and $350 \mu \mathrm{L}$ added immediately into each well of a 24 well plate before transfer to the incubator for $30 \min \left(37^{\circ} \mathrm{C}\right)$ to set the gel.

\subsection{Mechanical testing and morphological analysis of collagen hydrogels}

Collagen gels were formulated as in Section 2.3 at different collagen concentrations $(0.6,1.2,2.4$ and $3.0 \mathrm{mg} / \mathrm{mL}$ ). For mechanical testing, samples were placed in a servo-mechanic materials testing machine (TestResources 100-Q-225-6) fitted with a 0.5N capacity load cell. Samples were deformed to $0.15 \mathrm{~mm}$ at a rate of $0.02 \mathrm{~mm} / \mathrm{sec}$ with an $8.4 \mathrm{~mm}$ flat-ended indenter. ${ }^{19} \mathrm{~A}$ best-fit line was obtained from the force versus displacement data, giving the stiffness in $\mathrm{N} / \mathrm{mm}$. This stiffness was converted to Young's modulus E assuming the gel behaved as a thin elastic layer on a rigid substrate. ${ }^{19}$ For this, we used the method of Cao et al., ${ }^{20}$ assuming a value for the Poisson's ratio $v$ of 0.13 , typical for collagen gels. ${ }^{19}$ To assess the morphology of hydrogels, gels without cells were fixed in $2 \%$ glutaraldehyde [2 $\mathrm{h}$, room temperature (RT)] then processed for OTOTO-FESEM (Section 2.8).

\section{$2.5 \quad$ 2-D NSC culture on collagen hydrogels}

In preliminary experiments, the effects of altering density of collagen on NSC behaviour and the feasibility of imaging intraconstruct cells by FESEM were established. Here, dissociated NSCs were seeded on to the top of pre-formed collagen gels of different density $(0.6,1.2,2.4$ and $3.0 \mathrm{mg} / \mathrm{mL})$, in 24 well plates. NSCs were seeded at $3 \times 10^{4}$ cells $/ \mathrm{cm}^{2}$ in monolayer medium (herein termed ML-M [1:1 mix DMEM:F12, 2\% N2, $25 \mathrm{ng} / \mathrm{ml} \mathrm{EGF}$ and FGF, $5 \mathrm{ng} / \mathrm{ml}$ heparin, $50 \mathrm{U} / \mathrm{ml}$ penicillin and $50 \mathrm{\mu g} / \mathrm{ml}$ streptomycin]) and either cultured for 3-5 days (until confluent on glass) or changed to differentiation medium (neurosphere medium minus growth factors with the addition of $1 \%$ FBS) after $24 \mathrm{~h}$ and subsequently cultured for 7 days. Cells were then fixed in either $4 \%$ PFA (20 min, RT) for immunocytochemistry or $2 \%$ glutaraldehyde $(2 \mathrm{~h}, \mathrm{RT})$ for FESEM.

\subsection{Transfection of NSCs grown on collagen}

For subsequent experiments investigating magnetofection of NSCs grown on the surface of preformed collagen gels, NSCs were cultured on collagen gels of $0.6 \mathrm{mg} / \mathrm{mL}$. The day after plating, 
magnetofection complexes were formed by mixing $176 \mathrm{ng}$ pmaxGFP and $0.62 \mu \mathrm{L}$ Neuromag in base medium (1:1 mix of DMEM:F12) made up to a total volume of $75 \mu \mathrm{L}$. After 20 mins complex formation, $75 \mu \mathrm{L}$ was added to each well and plates were returned to the incubator and exposed to the desired magnetic field [no field, $\mathrm{F}=0 \mathrm{~Hz}$ (static field) and $\mathrm{F}=4 \mathrm{~Hz}$ (oscillating field)] for 30 mins. Controls had just DMEM:F12 addition. The plates were removed from the magnet and incubated for a further 30 mins before a full medium change to fresh ML-M medium. NSCs were fixed in 4\% PFA 48 h post-transfection to coincide with optimal GFP expression as previously reported. ${ }^{21}$ Safety assessments were also performed at this time-point. For an assessment of the differentiation potential of magnetofected NSCs grown on collagen, ML-M was changed to differentiation medium $24 \mathrm{~h}$ post-transfection. Cells were subsequently cultured for five days (with feeding every 2-3 days) and then fixed in 4\% PFA.

\subsection{LIVE/DEAD staining}

To assess NSC viability, cells were washed with PBS, incubated for $15 \mathrm{~min}\left(37^{\circ} \mathrm{C}\right)$ with $4 \mu \mathrm{M}$ calcein AM (produces green fluorescence in live cells) and $6 \mu \mathrm{M}$ ethidium homodimer-1 (produces red fluorescence in dead cells), washed again with PBS, then mounted for fluorescence microscopy.

\section{8 ОTOTO processing of collagen gels for FESEM}

A high resolution imaging technique termed ОTOTO-FESEM has been developed for imaging neural cells. ${ }^{9}$ Here, fixation and repeat staining of cells using osmium and thiocarbohydrazide can allow for detailed analysis of cellular membranes and polymeric materials with scanning electron microscopy. Glutaraldehyde fixed samples were washed three times in SCB (100 mM sodium cacodylate/2 mM calcium chloride, $\mathrm{pH} 7.2$ ) before post-fixation in a $1 \%$ osmium solution (RT, $1 \mathrm{~h}$ ). Samples were then sequentially stained with the high affinity osmium binding agent thiocarbohydrazide $(T)$ for 20 min then osmium ( 0 ) for $2 \mathrm{~h}$ (repeated twice) with six SCB washes between each step to obtain the OTOTO layering. Stained samples were dehydrated through a graded series of ethanols before critical point drying with liquid $\mathrm{CO}_{2}$ as the transition fluid. Samples were then mounted on scanning 
electron microscopy stubs with application of silver paint around the coverslip edges to enhance conductivity.

\subsection{Immunocytochemistry}

Immunostaining was performed on PFA fixed samples which were first incubated in blocking solution ( $5 \%$ normal donkey serum, $0.3 \%$ Triton-X in PBS) for $1 \mathrm{~h}$. This was replaced with primary antibodies, diluted in blocking solution, for $48 \mathrm{~h}\left(4^{\circ} \mathrm{C}\right)$ before washing three times in PBS. Primary antibodies (and dilutions) were for nestin (NSC cytoskeletal marker, 1:200), Sox-2 (NSC transcription factor, 1:1000), GFAP (astrocyte cytoskeletal protein, 1:250), Tuj-1 (neuronal cytoskeletal component, 1:500) and MBP (myelin constituent produced by oligodendrocytes, 1:100). Following washing, samples were incubated for a further $1 \mathrm{~h}$ in blocking solution before this was replaced by appropriate secondary antibodies also diluted in blocking solution (all at 1:200). These were incubated for $4 \mathrm{~h}$ at RT then washed three times in PBS with an elongated final washing step (at least $2 \mathrm{~h}$ ) before mounting using Vectashield mounting medium with DAPI.

\subsection{Imaging}

Fluorescence and phase microscopy: Fluorescence microscopy was performed using an AxioScope A1 microscope equipped with an Axio Cam ICc1 digital camera and AxioVision software (release 4.7.1, Carl Zeiss Microlmaging GmbH, Goettingen, Germany). Phase-contrast and fluorescence microscopy of live cells was performed using a Leica DM IL LED inverted microscope equipped with a FC420C digital camera and Leica Applications Suite software version 3.4.0 (Leica Microsystems, Wetzlar, Germany). Images were merged using Adobe Photoshop CS3 (version 10.0.1) prior to quantification. FESEM: Processed samples were examined using a Hitachi S4500 FESEM operated at 5kv accelerating voltage. 


\subsection{Assessment of transfection efficiency}

A microscopic method was chosen to analyse transfection efficiency of the NSCs grown on collagen. We have previously validated this approach when assessing nanoparticle mediated transfection efficiency in a range of neural cell populations including astrocytes, ${ }^{18} \mathrm{NSCs},{ }^{21-23}$ and oligodendrocyte precursor cells. ${ }^{24}$ This approach is crucial in enabling detailed analysis of both transfection outcome and safety (in terms of key cellular properties such as morphology, marker expression/profile and adherence) to be performed in parallel on the same samples. This is especially important in the context of examining the behaviour of transfected cells (including integration and migration within the matrix) in hydrogels which mimic biological environments. However, NSCs propagated on collagen form spheres over time which prevents quantification of transfection efficiency in terms of an absolute number of cells, as individual cells cannot be distinguished. Therefore, for a preliminary quantification of the efficiency of magnetofection protocols to transfect NSCs grown on collagen substrates, the proportions of transfected spheres was assessed in three double merged images taken at X100 magnification. To further quantify the extent of transfection, numbers of GFP positive cells per field were also counted. To quantify proportions of transfected astrocytes following magnetofection of NSCs and their differentiation on collagen, the same images used to assess neural marker expression were used to count the number of GFAP ${ }^{+}$cells, expressing GFP. This was expressed as a proportion of total $\mathrm{GFAP}^{+}$cells. This analysis was restricted to astrocytes as these cell types predominately retained expression of GFP after differentiation.

\subsection{Assessment of the safety of magnetofection of NSCs grown on collagen}

Safety was assessed by examining the effect of magnetofection procedures on key regenerative properties of the NSCs. First, numbers of spheres per field were counted and sphere diameter was measured across three images taken at X100 magnification; two parameters which are representative of the proliferative capacity of NSCs. Stemness was assessed by evaluating NSC marker expression in triple merged images (DAPI, GFP and appropriate neural marker; either nestin or Sox-2 for NSCs). Differentiation of NSCs, under all conditions, was assessed by examining triple 
merged images (X400) of cells stained with the appropriate neural marker. Proportions of daughter cells generated were counted and an assessment made of the cell types expressing GFP in at least three images ( $>100$ cells counted).

\subsection{Assessment of NSC membrane activity on collagen and glass}

In order to examine whether membrane activity of glass and collagen cultured NSCs is different, FESEM images of cell membranes (after culture for $3-5$ days on glass and $0.6 \mathrm{mg} / \mathrm{mL}$ collagen) were scored using a previously published 5-point analysis system for NSC membrane activity. ${ }^{9}$ Briefly, one field in the centre of each cell was analysed at X20000 magnification (corresponding to $25 \mu \mathrm{m}^{2}$ ) for extent of membrane ruffling, number of pits, number of filopodia and number of nanopodia. A count of the number of circular ruffles was also made over the whole cell. Ten cells were assessed for each condition over three separate cultures ( 30 cells glass; 30 cells for collagen).

\subsection{Statistical analysis}

All comparable data was analysed using Prism software (version 6.03, Graphpad). Data are presented as mean \pm standard error of the mean (SEM). For multiple groups statistical differences were measured by one-way ANOVA with Bonferroni's multiple comparison test (MCT). For comparison between two groups (i.e. the glass versus collagen membrane activity analysis) statistical differences were determined by an unpaired t-test. Repeat experiments (' $n$ ') are using cells derived from a different mouse litter.

\section{Results}

\subsection{Biomimetic substrates can be formulated using collagen}

Fibrous, ECM-like matrices were formulated using various concentrations of collagen with clear differences observed in mesh formation and pore size between 0.6 and $3.0 \mathrm{mg} / \mathrm{mL}$ collagen concentrations (Figure 1A and B). Increasing the concentration of collagen resulted in increasing 
Young's modulus of the samples from $34 \pm 5$ to $350 \pm 50$ Pa for $0.6 \mathrm{mg} / \mathrm{mL}$ to $3.0 \mathrm{mg} / \mathrm{mL}$ respectively (force versus displacement slope and Young's modulus for the hydrogels formed using each collagen concentration are shown in Figure 1C).
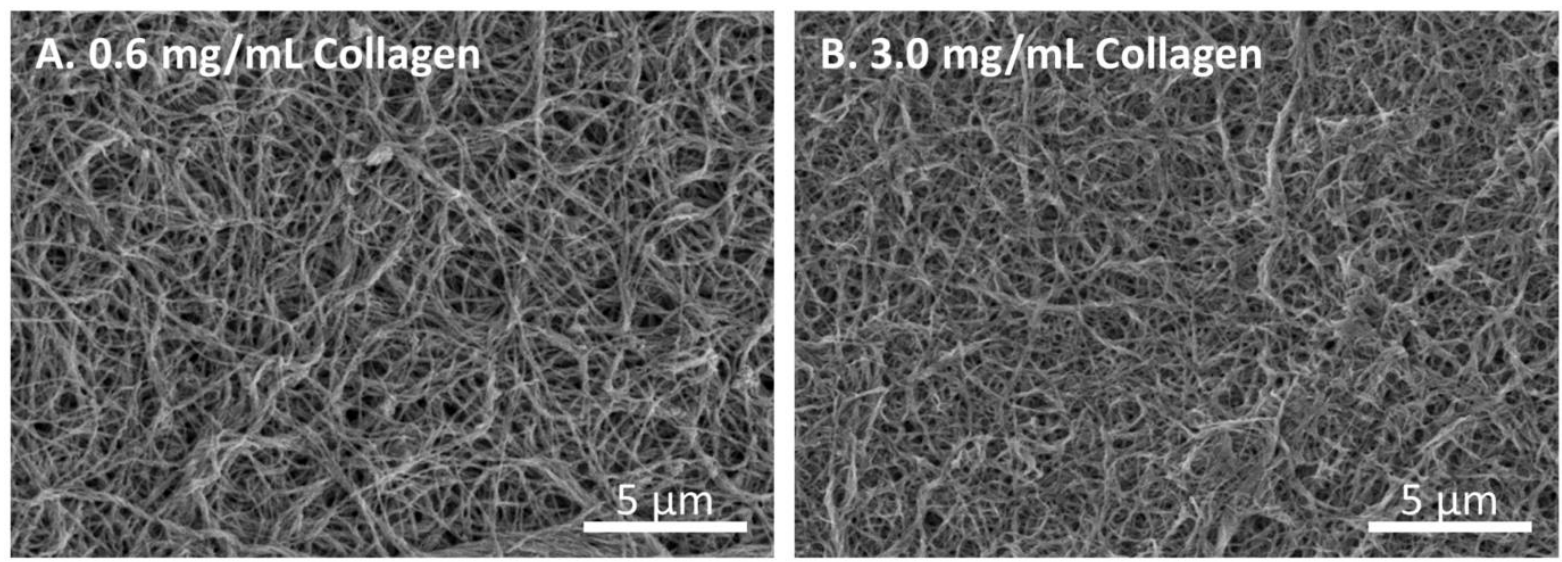

\begin{tabular}{lll} 
C. Concentration $(\mathrm{mg} / \mathrm{ml})$ & Slope $(\mathrm{N} / \mathrm{mm})$ & $\mathrm{E}(\mathrm{Pa})$ \\
\hline 0.6 & $0.0060 \pm 0.0008$ & $34 \pm 5$ \\
1.2 & $0.026 \pm 0.002$ & $150 \pm 10$ \\
2.4 & $0.044 \pm 0.003$ & $240 \pm 20$ \\
3.0 & $0.062 \pm 0.096$ & $350 \pm 50$
\end{tabular}

Figure 1. Morphological and physical characterisation of collagen hydrogels. (A-B) Representative FESEM images of (A) $0.6 \mathrm{mg} / \mathrm{mL}$ and $(B) 3.0 \mathrm{mg} / \mathrm{mL}$ collagen hydrogels showing the fibrous ECM-like nature of the polymer and differences in mesh formation and pore size between the two collagen concentrations. (C) Table showing force versus displacement slope and Young's modulus, E, of hydrogels made with increasing concentrations of collagen $(n=3)$.

\subsection{NSCs can be successfully propagated and differentiated on collagen matrices}

NSCs were successfully propagated on pre-formed collagen gels of varying density $(0.6-3.0 \mathrm{mg} / \mathrm{mL})$. Normal, intact and circular nuclei were observed in the cells with the majority of cells positive for the NSC marker nestin, suggesting the cells are healthy and retain a stem cell phenotype (Figure $\mathbf{2 A}$ and B). Across all collagen densities, NSCs demonstrated a propensity to form spheres of aggregated cells, with this phenomenon most apparent when NSCs were cultured on the highest density collagen matrix, $3.0 \mathrm{mg} / \mathrm{mL}$ (Figure 2B). Pairs of cells appearing to result from a recent division were 
clearly observed by OTOTO-FESEM suggesting the NSCS retain the ability to proliferate (Figure 2A-

inset). Spheres of cells were also frequently observed (Figure 2B - inset), confirming the finding

from fluorescence microscopy that the NSCs tend to proliferate as spheres attached to the collagen.

The majority of NSCs displayed LIVE (green) staining after culture on collagen (estimated to be over 90\%, although individual cells were not distinguishable), with DEAD (red) cells appearing around the edges of the formed spheres (Figure 2C).
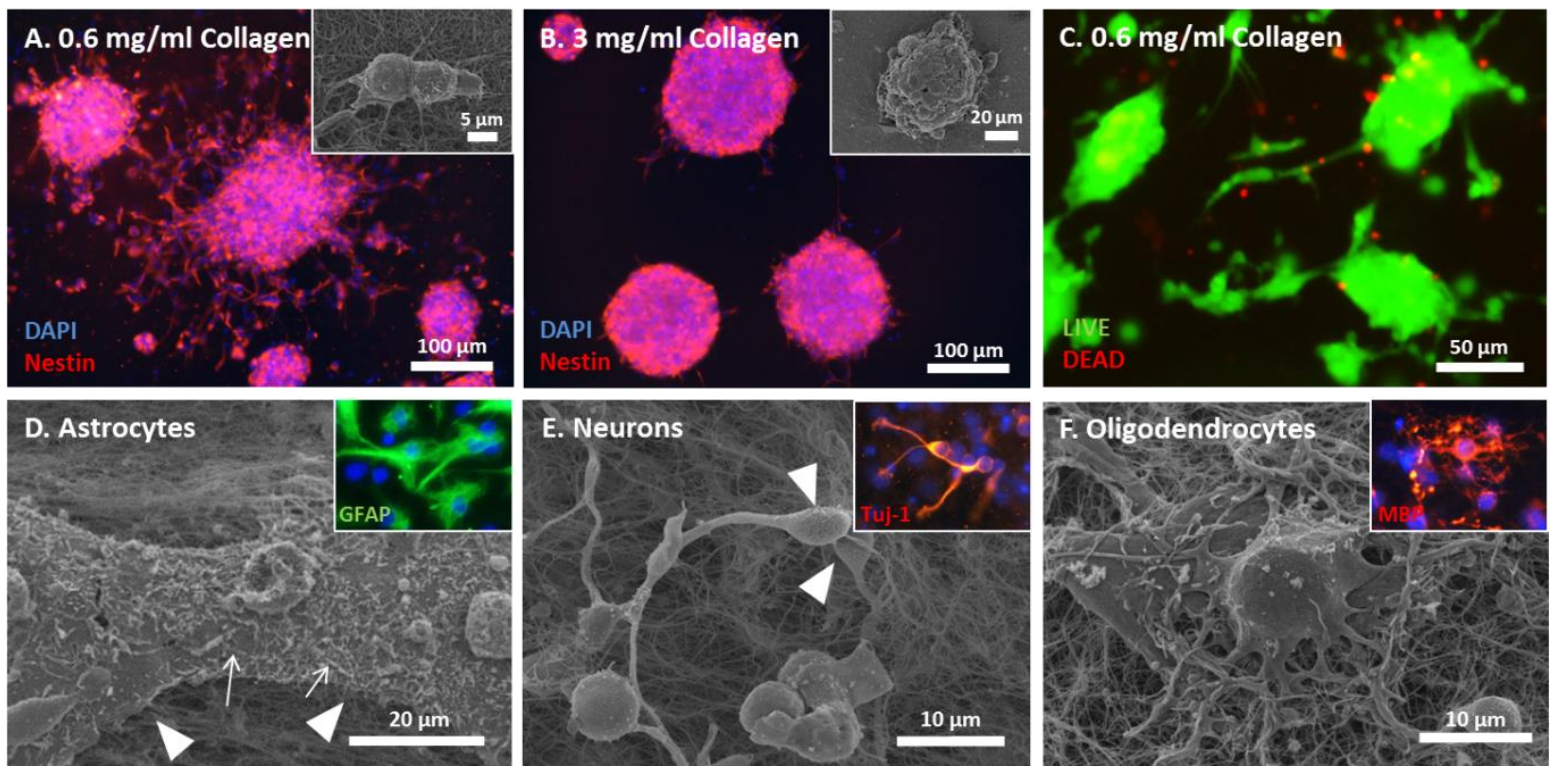

Figure 2. NSC propagation and differentiation on collagen. (A and B) Representative fluorescent images of NSCs propagated for 4 days on (A) $0.6 \mathrm{mg} / \mathrm{mL}$ and (B) $3.0 \mathrm{mg} / \mathrm{mL}$ density collagen. Note that more cellular migration from the sphere seems to be apparent when NSCs are cultured on the lower density collagen. ( $A$ and $B$ insets) FESEM image of ( $A$ inset) dividing NSCs and ( $B$ inset) NSCS forming a neurosphere after propagation on collagen. (C) LIVE/DEAD staining of NSCs grown on collagen. (D-F) Representative FESEM images following NSC differentiation on collagen of (D) astrocytes, $(E)$ neurons and $(F)$ oligodendrocytes. Arrow heads in $(D)$ indicate two astrocytes after recent division and in (E) indicate neurons. Note membrane features such as filopodia can be readily identified (arrows in D) and cell protrusions appear to be growing underneath collagen fibrils (especially apparent in E). (D-F insets) Fluorescence images of respective daughter cells generated by differentiating NSCs on collagen. 
NSCs grown on collagen could be successfully differentiated into all the daughter cell types; astrocytes, neurons and oligodendrocytes (Figure 2D-F). These were generated in similar proportions to those differentiated on glass (quantification performed in Figure 4) with broadly similar characteristic morphologies (Figure 2D-F). High magnification imaging using OTOTO-FESEM showed some evidence of cell integration/migration into the collagen matrix, especially evident in the case of neurons that appeared to be extending processes below the surface layer of collagen (Figure 2E). Membrane features were straightforward to identify and a clear difference can be noted between the astrocytes, which elaborate numerous filopodia, and the neurons/oligodendrocytes, which appear to be relatively quiescent with less evidence of membrane activity (Figure 2D-F).

\subsection{Magnetic field application could safely enhance MNP mediated gene delivery to NSCs} grown on collagen gels

To investigate the feasibility of MNP mediated gene delivery to cells in collagen gels, NSCs were propagated on gels with collagen density of $0.6 \mathrm{mg} / \mathrm{mL}$. At this stiffness it was observed that more single cells were present than on $3.0 \mathrm{mg} / \mathrm{mL}$ thereby reducing physical constraints to MNP access to cells which are inside spheres. After transfection, GFP expression was observed in nestin positive cells within spheres that had formed on the collagen (Figure 3A). All spheres observed microscopically were nestin or Sox-2 positive and all transfected cells were also nestin or Sox-2 positive (Figure 3A-B), suggesting magnetofection protocols have limited or no effect on stem cell marker expression. Magnetofected NSCs are capable of division with recently divided, nestinpositive NSCs, both expressing GFP shown in Figure 3C. Microscopic analysis of the NSCs revealed apparently higher numbers of GFP positive cells per field and greater levels of GFP expression within neurospheres after adding magnetofection complexes in the presence of magnetic fields compared to no-field (Figure 3A). Quantification of the percentage of spheres displaying transfection under a no-field condition revealed that basal levels were $18.6 \pm 5.7 \%$. This was significantly enhanced and 
almost tripled when transfection was performed in the presence of a static $(46.1 \pm 4.9 \%)$ and an oscillating ( $50.3 \pm 3.5 \%)$ magnetic field (Figure 3D). Application of an oscillating field also significantly enhanced the number GFP expressing cells per field compared to no-field $(17.2 \pm 3.6$ versus $7.1 \pm 0.5$ respectively; Figure 3E).

Following magnetofection of NSCs grown on collagen, there were no differences in sphere number or size across all conditions (Figure 3F-G). Using a LIVE/DEAD stain, high NSC viability was observed after oscillating field magnetofection (estimated to be above 90\%) which was similar to control cells where no particles were added (data not shown). 

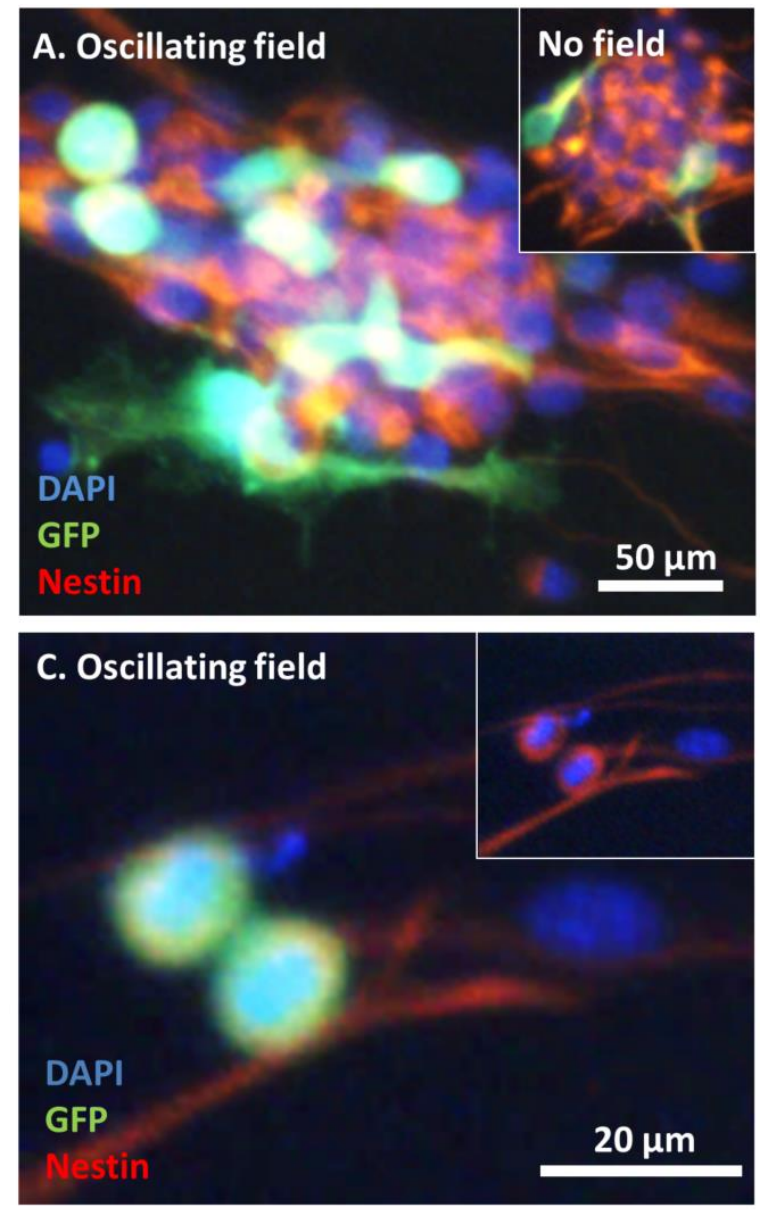

E. Number of transfected cells per field

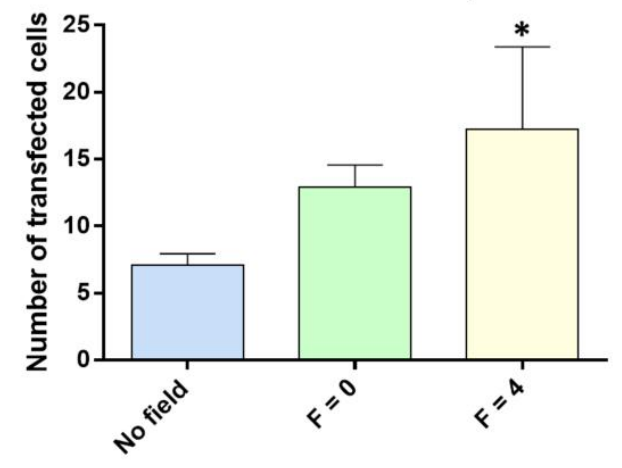

Field condition

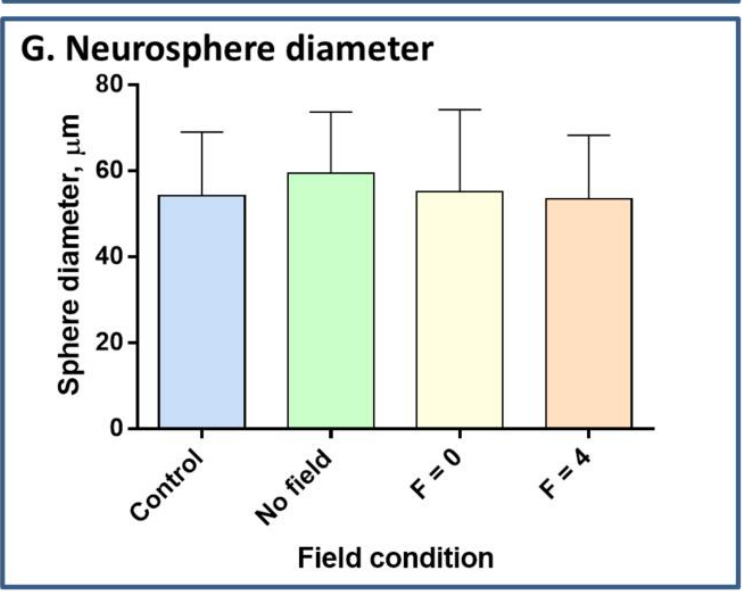

G. Neurosphere diameter

\section{B. Oscillating field}

8

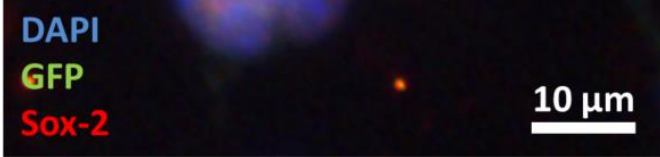

D. Proportion of transfected spheres
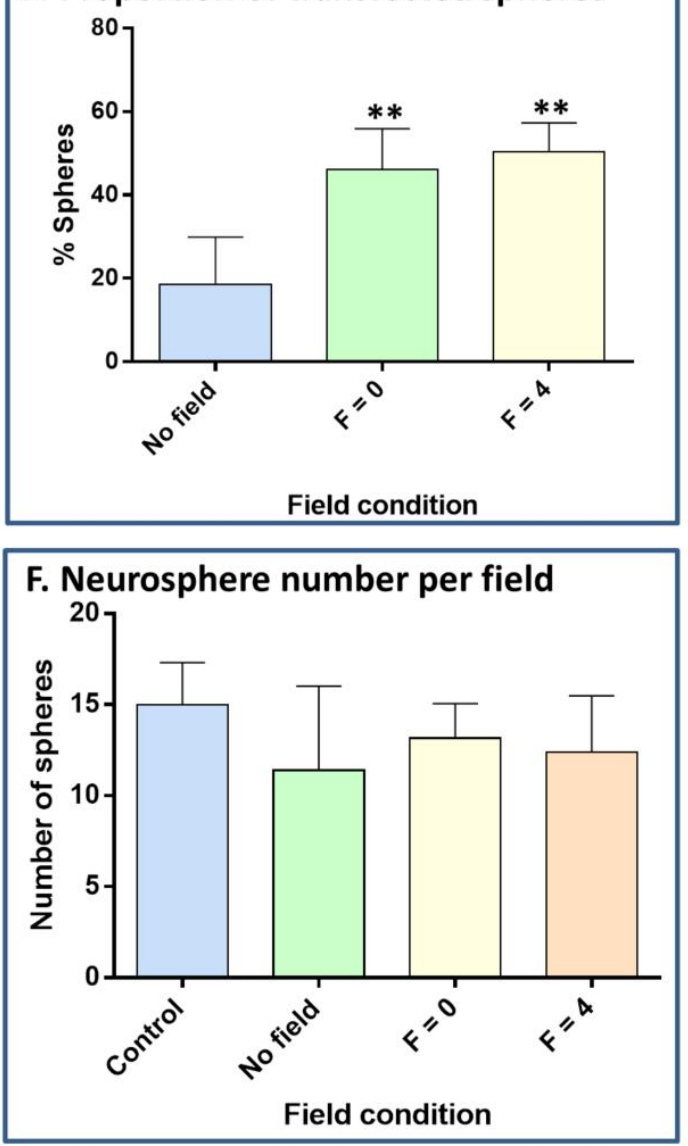
Figure 3. Field application safely enhances MNP mediated gene delivery in NSCs grown on collagen gels. Representative images of NSCs grown on $0.6 \mathrm{mg} / \mathrm{mL}$ collagen and transfected under $(A) F=4 \mathrm{~Hz}$ oscillating magnetic field or ( $A$, inset) no field. In $(A)$ it can also be noted that spheres remain positive for NSC marker nestin. (B) Representative triple merged, fluorescent image of a transfected sphere positive for NSC marker Sox-2. (C) Representative triple merged image of two nestin positive transfected cells formed from a recent division with inset showing the nestin staining minus GFP expression. (D-E) Bar charts depicting (D) proportions of transfected spheres and $(E)$ number of transfected cells per field across the different transfection conditions. (F-G) Bar charts depicting (F) numbers of neurospheres per field and (G) sphere diameter after transfection of NSCs on collagen across all conditions. Significant differences are ${ }^{* *} p<0.01$ and ${ }^{*} p<0.05$ vs no-field (one-way ANOVA and Bonferroni's MCT, $n=4)$.

\subsection{Magnetofected NSCs differentiated normally on collagen}

After transplantation, NSCs can mediate their therapeutic effect by differentiation into their progeny for cell replacement. Therefore, it is important to assess the expression of the introduced gene in the daughter cells and any effects of the gene engineering strategies on the differentiation profile of NSCs. The ability of NSCs to differentiate into their daughter cells on collagen was not affected by magnetofection protocols, with astrocytes, neurons and oligodendrocytes all generated by the engineered NSCs (Figure 4A-C). Daughter cells were also produced in similar proportions across all conditions indicating magnetofection has no effect on the differentiation profile of NSCs (Figure 4DF). The majority of transfected cells were astrocytes with small numbers $(\mathrm{ca}<3 \%)$ of transfected neurons observed. The proportions of GFP expressing astrocytes was estimated to be $1.3 \pm 0.7 \%, 2.5$ $\pm 1.3 \%$ and $3.3 \pm 0.4 \%$ for no field, static field and the $\mathrm{F}=4 \mathrm{~Hz}$ oscillating field respectively. This pattern (no-field $<\mathrm{F}=0 \mathrm{~Hz}<\mathrm{F}=4 \mathrm{~Hz}$ ) is similar to the pattern of numbers of transfected cells 
counted per field in the parental NSCS (Figure 3E). However, no significant differences were found in the levels of transfected astrocytes between field conditions.

A. Astrocytes

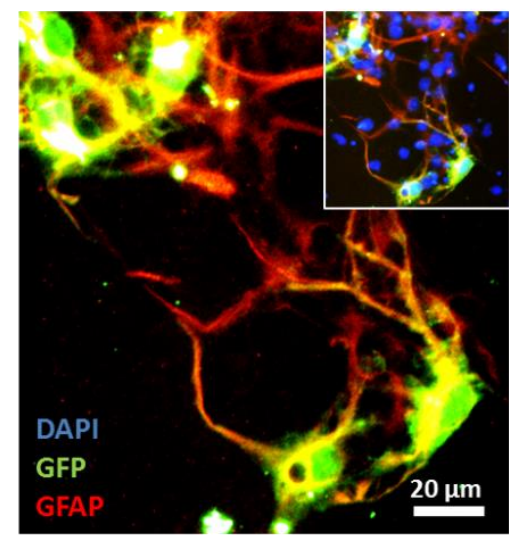

D. Astrocytes

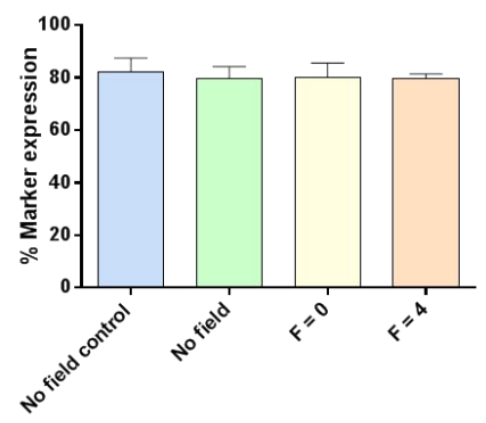

Field condition
B. Neurons

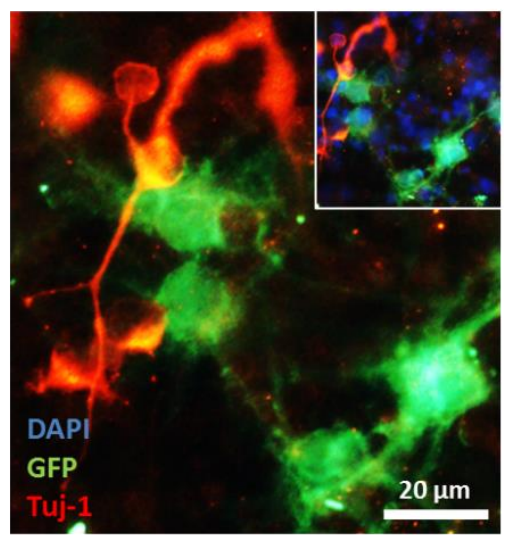

E. Neurons

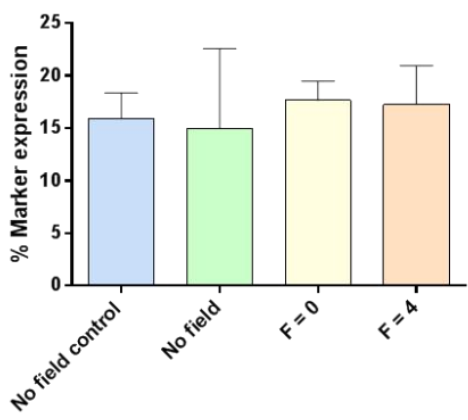

Field condition
C. Oligodendrocytes

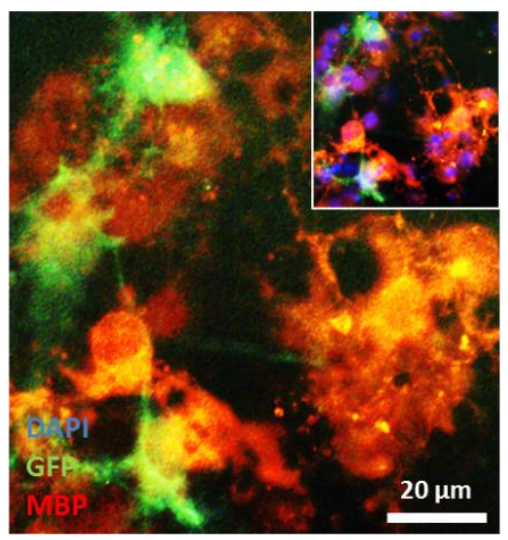

F. Oligodendrocytes

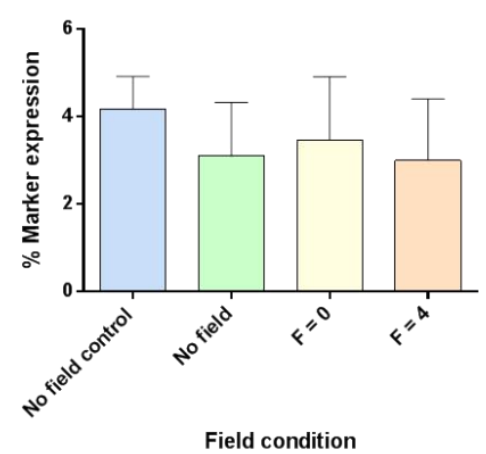

Figure 4. Differentiation of magnetofected NSCs on collagen. Representative double merged

fluorescent images of (A) astrocytes, (B) neurons and (C) oligodendrocytes generated from NSCs transfected under the $\mathrm{F}=4 \mathrm{~Hz}$ condition and allowed to differentiate on collagen. (Insets) Identical fields to $A, B$ and $C$ with addition of DAPI. Bar charts displaying quantification of the proportions of (D) astrocytes, (E) neurons and (F) oligodendrocytes generated under all tested conditions.

\subsection{Assessment of NSC membrane activity when grown on glass or collagen}

Using OTOTO-FESEM, NSC membrane features (including filopodia, pits, nanopodia and circular ruffles) were clearly identified (Figure 5A). The membranes of NSCs cultured on collagen appeared relatively quiescent and 'less active' than the membranes of NSCs grown on glass in terms of the 
extent of expression of these features. To quantify any potential differences, the extent of membrane activity of NSCs grown on the two substrates was assessed using a previously developed 5-point classification system for membrane features. ${ }^{9}$ In this assessment, four out of the five markers of membrane activity (extent of membrane ruffling and numbers of pits, filopodia and nanopodia) were significantly elevated in NSCs grown on glass when compared to the NSCs grown on collagen (Figure 5B-E). A tendency towards increased numbers of circular ruffles on the membranes of glass cultured NSCs was also noted but was not found to be significantly different (Figure 5F).

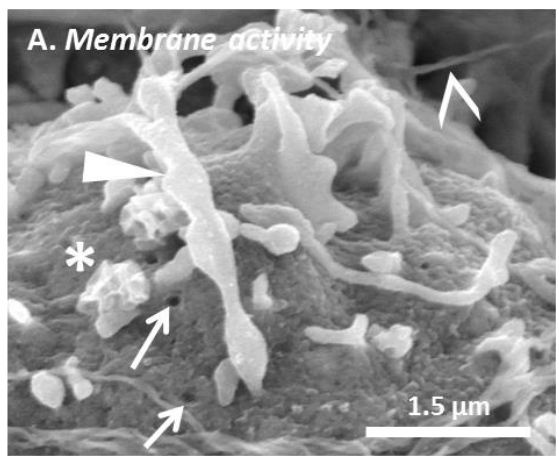

B. Membrane ruffling

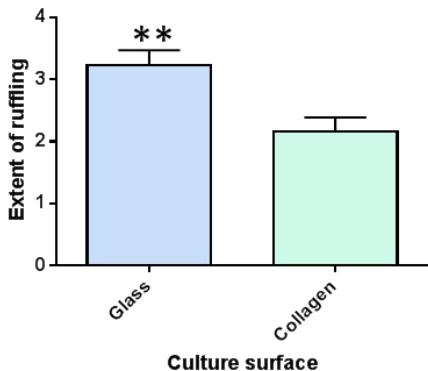

E. Numbers of nanopodia

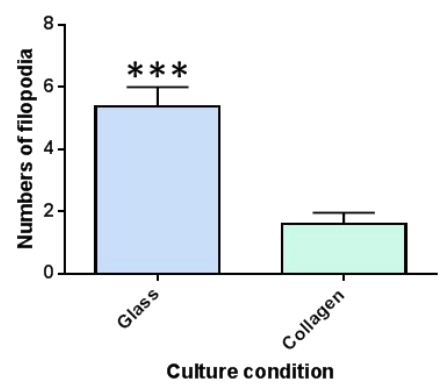

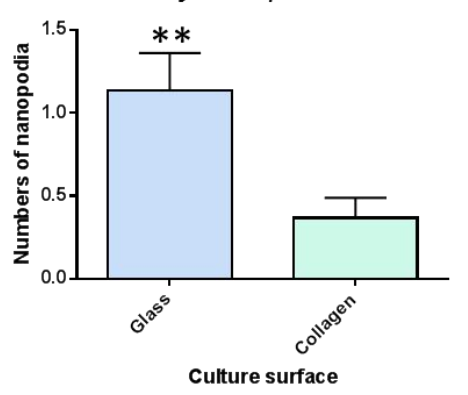

C. Number of pits

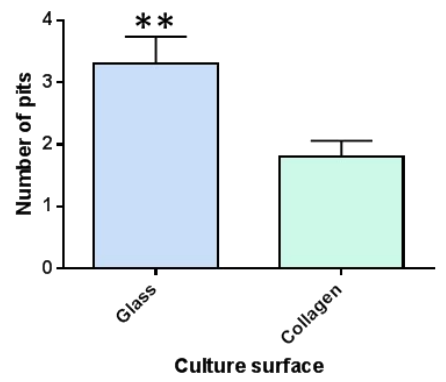

F. Numbers of circular ruffles

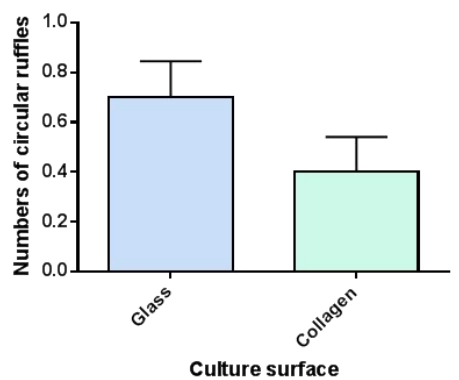

Figure 5. Quantification of membrane features of NSCs propagated on glass or collagen. (A)

Representative FESEM image of NSC membrane depicting filopodia (block arrowhead), nanopodia (empty arrowhead), pits (arrows) and circular ruffles (adjacent to asterisk). (B-F) Bar charts displaying quantification of various membrane features highlighted in the figure. Statistical differences are ${ }^{* *} p<0.01$ and ${ }^{* * *} p<0.001$ as evaluated by an unpaired $t$-test. 


\section{Discussion}

Tissue engineering studies across different physiological systems are currently witnessing a paradigm shift to the experimental culture of cells in more biologically relevant, and biomimetic 3D structures compared with the artificial 2D cultures on hard substrates which represent the status quo. Major examples of this include replicating key physiological behaviour of hepatocytes in $3 D,{ }^{25}$ to facilitate accurate reflection of drug metabolism by the liver, and recreating cancer cell behaviour for superior modelling of anti-tumour drug efficacy and resistance. ${ }^{26,27}$ This approach is still in its infancy with respect to 3D cultures of neural cells. However, key neural cell properties such as survival, differentiation and cytoarchitectecture have been shown to be more in vivo like in 3D neural cultures than in $2 \mathrm{D}$ cultures. ${ }^{28}$ As cell physiology and physical structure govern nanomaterial interaction with neural tissue it is essential that future experiments use such models to more accurately predict functionality and toxicity of novel nanotherapeutics. Indeed, such systems can be developed using human cells ${ }^{29}$ therefore potentially offering crucial pre-clinical models of greater translational relevance than the current gold standard animal models.

As far as we are aware, this is the first time that primary NSCs propagated on a soft hydrogel matrix have been genetically engineered in situ. Specifically, a nanoparticle based vector system in conjunction with magnetofection technology has never been used for this purpose. As transfection efficiency using nanoparticle methodologies is governed by endocytotic activity, a high-resolution FESEM based imaging technique was also utilised to examine membrane activity of NSCs grown on hydrogels, constituting a novel and critical application for this technique in terms of elucidating nanomaterial interactions with transplant cells within such 'soft' substrates. Nanoparticle systems are being widely commercialised as biomedical tools due to their multifunctionality and good safety profile, however, these have not previously been used to engineer neural cells propagated in hydrogel matrices, nor has their compatibility with magnetofection technology been evaluated to date. This is a crucial extension of nanovector platforms, as hydrogel based materials are a globally emergent technology for cellular research in tissue mimetic 3D constructs, widely predicted to 
enable measurement of more biologically relevant outputs than 2D culture. Accordingly, we consider that the data presented in this study provide important findings for the neurobiology, neurotransplantation and regenerative medicine fields.

This is the first study testing oscillating field magnetofection in cells propagated using soft hydrogel materials. Although it is not fully understood how an oscillating magnetic field promotes MNP mediated transfection efficiency, it has been proposed that it may be due to mechanical membrane stimulation which enhances endocytosis of MNP:DNA complexes. ${ }^{30}$ Here, cells were grown on the hydrogel surface, increasing cell distance from the magnet positioned below the culture plate. Magnetic force acting on a particle decreases inversely with the fifth power of distance from the source $\left(F_{\text {mag }} \approx 1 / r^{5} ; F_{\text {mag }}=\right.$ Magnetic force and $r=$ distance from source $) .{ }^{31}$ Therefore, oscillating magnetofection efficiency decreases with increasing cell distance from the magnet. ${ }^{30}$ Increased distances, combined with the low levels of membrane activity detected on NSCs grown with hydrogels, could account for the lack of an effect generated by oscillating magnetic fields (over static fields) observed in this study.

However, for a more clinically and biologically relevant 3D matrix, transplant cells will need to be engineered throughout the depth of the construct, and the efficacy of our approach needs to be tested using 3D cell functionalised hydrogels. There are precedents suggesting that an 'intraconstruct-cell engineering' approach for this purpose may be entirely feasible. As an example, others have shown the feasibility of MNP penetration into collagen hydrogel matrices, with MNPs (diameter: $100 \mathrm{~nm}$ ) penetrating the gels up to depths of $800 \mu \mathrm{m}$ when under application of a static magnetic field for $22 \mathrm{~h} .^{32}$ In particular, 3D transfection of non-neural NIH-3T3 cells (a fibroblastic cell line) using MNPs (diameter: ca. $36 \mathrm{~nm}$ ) and application of a static magnetic field for $3 \mathrm{~h}$ was demonstrated, with an efficiency of approximately $75 \%$ up to a depth of $2 \mathrm{~mm} .{ }^{33}$ Therefore, taking our data together with such 3D particle engineering studies, it can be suggested that intraconstruct 
neural cell engineering using MNPs for clinical application may be feasible and warrants future examination.

In terms of the physiological relevance of the model described, neurospheres have been used to investigate NSC biology and they can recapitulate some of the physiological parameters of the in vivo stem cell niche, including in maintaining cell-cell contacts and NSC developmental stage. ${ }^{34}$ Although collagen (as used in this report) is rarely found in the CNS, it has been used for many neural applications, including implantation with NSCs into sites of injury and disease. ${ }^{35}$ Importantly for mimicking native NSC environments, collagen hydrogels replicate the mechanically soft nature of the brain. Matching stiffness to specific NSC niche regions in the brain is critical as it has been shown that cells respond to physical cues (e.g. altering their proliferation or differentiation profiles) in a process termed mechanotransduction. ${ }^{36}$ In particular, NSCs have been shown to alter their proliferation, migration and fate in response to stiffness changes ${ }^{12-15}$ and this has led to speculation that mechanical properties may play a role in maintaining spatially defined populations of cells within the brain. Supporting this concept, Iwashita et al demonstrated clear spatiotemporal changes in stiffness within the developing mouse cerebral cortex, suggesting that changing mechanical properties may indeed influence the maintenance of the different cerebral cortex layers, including the ventricular zone (VZ) and SVZ NSC niches. ${ }^{37}$ We observed that, over the range of collagen concentrations used here, hydrogel stiffness increased with collagen concentration from ca. 34 to $350 \mathrm{~Pa}$. The value of the gel stiffness in our work was in agreement with that found by Raub et al. using a similar method, who found a Young's modulus of $300 \mathrm{~Pa}$ at a concentration of $3.0 \mathrm{mg} / \mathrm{mL}^{19}$ Our measurements are within the wide range of stiffnesses reported for embryonic to adult brain (ca. $30-3000 \mathrm{~Pa}$ ). ${ }^{37-39}$ However, our stiffness measurement at $0.6 \mathrm{mg} / \mathrm{mL}$ (ca. $34 \mathrm{~Pa}$ ) is similar to that of the embryonic VZ and SVZ (ca. $50-75$ Pa at E12.5 - 14.5) ${ }^{37}$ which is particularly advantageous for replicating the mechanical properties of the NSC niche during a period in which NSCs are undergoing expansive proliferation and differentiation (to populate the developing brain). ${ }^{40}$ NSCs appear at about E8 within the VZ in the rodent embryonic brain. ${ }^{40}$ As development proceeds 
these cells start to populate and form a distinct SVZ region (from E10 onwards) ${ }^{41}$ and a residual population of NSCs is maintained within the VZ and SVZ. From the SVZ, NSC migrate into the outer layers of the cerebral cortex (including the intermediate zones and cortical plate) where they differentiate into neurons and glial cells. ${ }^{40,41}$ Therefore, our gels may have biomimetic utility in matching tissue stiffness of active, early embryonic NSC niches. Collagen has also been used in several studies to mimic ECM structures, for example, when investigating cancer cell invasion through $\mathrm{ECM}^{42}$ and cells within 3D ECM-like collagen hydrogels have been shown to behave more like their in vivo counterparts. ${ }^{43}$ Crucially, collagen is transparent and therefore facilitates live cell monitoring of intraconstruct cells using both phase and fluorescence imaging technologies. Therefore, this system could be used for future mechanistic analysis of gene delivery technologies (in a biomimetic substrate) using sophisticated imaging modalities such as dynamic time-lapse microscopy or fluorescence resonance energy transfer. ${ }^{17}$

There is also high potential to enhance cellular complexity by using collagen as a biomimetic substrate. For example, external differentiated cells and other ECM signalling molecules also play a part in maintaining the NSC niche. ${ }^{34}$ Future work may be able to more closely replicate the NSC niche by modifying the collagen with known molecules important in stem cell biology (such as laminin) ${ }^{40}$ and potentially the inclusion of surrounding cell types, such as astrocytes. There is also a need to investigate the versatility of the protocols in a range of hydrogel formulations. For example, selfassembling peptides offer substantial benefits in replicating CNS microenvironments including biocompatibility, tight control over mechanical properties, such as stiffness and degradation and the ability to modify chemical motifs to replicate those on native ECM. ${ }^{44}$

Predicting cell responses and amenability to magnetofection protocols when grown on/within soft substrates is not straightforward as neural cell behaviour, such as migration and differentiation, has been shown to significantly change on softer substrates. ${ }^{12-14,45}$ In addition, endocytosis, which is required for nanoparticle uptake and transfection, has also been shown to be reduced in cells grown 
on soft compared to hard substrates. ${ }^{16,17}$ Of wide interest to the nanobiotechnology community, we therefore present a high resolution imaging technology for examining cellular membranes and interactions with polymer matrices to allow assessment of material biocompatibility and a range of parameters indicating cell behaviour. Here we show for the first time that an ОТОТО fixation methodology for FESEM is compatible with hydrogel culture of NSCs, a highly novel approach in the observation of neural cells grown with hydrogels. ОТОТО fixation facilitates visualisation of cellular features and nanofibrils (which constitute the collagen hydrogel) by FESEM at resolutions far superior to light microscopy and offers improvement over conventional scanning electron microscopy as it avoids the use of gold sputter coating which can mask membrane features. ${ }^{9}$ In addition, OTOTO-FESEM provides higher throughput sample processing and analysis than transmission electron microscopy (TEM) as numerous samples can be processed in parallel and whole areas of membrane on multiple cells can be analysed quickly (compared to the analysis of small sections of a single cell membrane in TEM). ${ }^{9}$ Combining FESEM with backscatter electron detection of iron oxide within MNPs is also feasible ${ }^{9}$ and could allow future analysis of MNP interaction with important membrane features of intraconstruct cells, providing information on optimal particle design for cellular uptake.

In our study, several membrane features (membrane ruffling, filopodia, pits, nanopodia and circular ruffles) were identified using OTOTO-FESEM both of cells grown on glass and collagen. These were identical to features previously identified on NSCs and suggested to be predictors of nanoparticle trafficking and uptake. ${ }^{9}$ For example, filopodia and nanopodia sense the surrounding environment ${ }^{47}$ and therefore facilitate potential detection of extracellular particles, pits are involved with MNP endocytosis ${ }^{47}$ and, although the function of circular ruffles is currently unknown, they may play a part in micropinocytosis. ${ }^{48}$ Interestingly, these all displayed evidence of reduced activity in NSCs which were grown on collagen compared to glass, except for circular ruffles. Other studies have shown endocytosis to be reduced on soft substrates compared to hard. ${ }^{16,17}$ Therefore, a reasonable explanation for the lower levels of these membrane features could be a reduction in endocytotic 
activity in NSCs grown on collagen compared to glass. A direct comparison of transfection/particle uptake efficiency of magnetofection in NSCs grown on collagen or glass would have to be performed to confirm this. However, reduced endocytosis, and therefore MNP uptake, in NSCs grown on soft substrates could be a major barrier to using magnetofection technology for transfection and labelling of NSCs in physiological environments. A combination of high resolution FESEM and timelapse microscopy may be used in future experiments to elucidate endocytotic mechanisms/dynamics of NSCs grown on collagen to determine whether MNP engineering of intraconstruct cells is viable.

\section{Conclusions}

We have presented data that demonstrate for the first time that therapeutically important nanomedicine technologies can be tested in cells propagated using in vitro systems which more closely represent the physiological tissue they are derived from than standard 2D cellular cultures. We show that magnetofection is a safe methodology to genetically engineer NSCs grown using such systems offering promise for the future translation of the technology; although there is a marked reduction in membrane activity of cells grown on soft compared to hard substrates. The latter finding could suggest a barrier to MNP mediated cell engineering approaches, which rely on membrane uptake, in these neuromimetic environments. However, using such techniques as we have described, magnetofection protocols and particle design may be improved to overcome such a challenge in a neuromimetic context and further could have wider application to testing nanotechnologies in a range of physiologically relevant systems.

\section{Acknowledgements}


The authors wish to acknowledge the expert contribution of Professor David Furness (Keele

University) who assisted with the FESEM image capture and analysis. The work was funded by the Engineering and Physical Sciences Research Council.

\section{References}

1 O. Lindvall and Z. Kokaia, J. Clin. Invest., 2010, 120, 29.

2 A. Trounson and C. Mcdonald, Cell Stem Cell, 2015, 17, 11-22.

3 C. Plank, O. Zelphati and O. Mykhaylyk, Adv. Drug Deliv. Rev., 2011, 63, 1300-1331.

4 M. R. Pickard, C. F. Adams, P. Barraud and D. M. Chari, J. Funct. Biomater., 2015, 6, 259-76.

5 C. F. Adams, L. L. Israel, S. Ostrovsky, A. Taylor, H. Poptani, J.-P. Lellouche and D. M. Chari, Adv. Healthc. Mater., 2016, 5, 841-9.

6 M. Song, Y.-J. Kim, Y. Kim, J. Roh, S. U. Kim and B.-W. Yoon, Hum. Gene Ther., 2010, 21, 60310.

7 C. F. Adams, M. R. Pickard and D. M. Chari, Nanomedicine: NBM, 2013, 9, 737-741.

8 M. Neri, C. Maderna, C. Cavazzin, V. Deidda-Vigoriti, L. S. Politi, G. Scotti, P. Marzola, A. Sbarbati, A. L. Vescovi and A. Gritti, Stem Cells, 2008, 26, 505-516.

9 A. R. Fernandes, C. F. Adams, D. N. Furness and D. M. Chari, Part. Part. Syst. Charact., 2015, 32, 661-667.

10 F. Gattazzo, A. Urciuolo and P. Bonaldo, Biochim. Biophys. Acta-Gen. Subj., 2014, 1840, 2506-2519.

11 M. M. Pakulska, B. G. Ballios and M. S. Shoichet, Biomed. Mater., 2012, 7, 024101.

12 N. D. Leipzig and M. S. Shoichet, Biomaterials, 2009, 30, 6867-78.

13 A. J. Keung, E. M. De Juan-Pardo, D. V. Schaffer and S. Kumar, Stem Cells, 2011, 29, 1886-97.

14 K. Saha, A. J. Keung, E. F. Irwin, Y. Li, L. Little, D. V Schaffer and K. E. Healy, Biophys. J., 2008, 95, 4426-38.

15 H. Mori, A. Takahashi, A. Horimoto and M. Hara, Neurosci. Lett., 2013, 555, 1-6.

16 C. Huang, P. J. Butler, S. Tong, H. S. Muddana, G. Bao and S. Zhang, Nano Lett., 2013, 13, 1611-5.

17 H. J. Kong, J. Liu, K. Riddle, T. Matsumoto, K. Leach and D. J. Mooney, Nat. Mater., 2005, 4, 460-464.

18 M. R. Pickard and D. M. Chari, Nanomedicine (Lond), 2010, 5, 217-232.

19 C. B. Raub, A. J. Putnam, B. J. Tromberg, S. C. George, Acta Biomater., 2010, 6, 4657-65. 
A. R. Fernandes and D. M. Chari, J. Control. Release, 2016, Part I.

A. R. Fernandes and D. M. Chari, J. Control. Release, 2016, Part II.

S. I. Jenkins, M. R. Pickard, N. Granger and D. M. Chari, ACS Nano, 2011, 5, 6527-6538.

R. Kostadinova, F. Boess, D. Applegate, L. Suter, T. Weiser, T. Singer, B. Naughton and A. Roth, Toxicol. Appl. Pharmacol., 2013, 268, 1-16.

D. D. Pearse, A. R. Sanchez, F. C. Pereira, C. M. Andrade, R. Puzis, Y. Pressman, K. Golden, B. M. Kitay, B. Blits, P. M. Wood and M. B. Bunge, Glia, 2007, 55, 976-1000. Lawrenson, Lab. Invest., 2013, 93, 528-42.

M. C. Laplaca, V. N. Vernekar, J. T. Shoemaker and D. K. Cullen, in Methods in Bioengineering: 3D Tissue Engineering, ed. F. Berthiaume, J. R. Morgan, Artech House, Norwood, MA, 2010, Chapter 11, 187-204.

S. H. Choi, Y. H. Kim, M. Hebisch, C. Sliwinski, S. Lee, C. D’Avanzo, H. Chen, B. Hooli, C. Asselin, J. Muffat, J. B. Klee, C. Zhang, B. J. Wainger, M. Peitz, D. M. Kovacs, C. J. Woolf, S. L. Wagner, R. E. Tanzi and D. Y. Kim, Nature, 2014, 515, 274-8.

A. Fouriki, N. Farrow, M. A. Clements and J. Dobson, Nano Rev., 2010, 1, 1-5.

B. Polyak, I. Fishbein, M. Chorny, I. Alferiev, D. Williams, B. Yellen, G. Friedman and R. J. Levy, Proc. Natl. Acad. Sci. U. S. A., 2008, 105, 698-703.

H. W. Child, P. A. Del Pino, J. De La Fuenta, A. S. Hursthouse, D. Stirling, M. Mullen, G. M. McPhee, C. Nixon, V. Jayawarna and C. C. Berry, ACS Nano, 2011, 5, 7910-9.

H. Zhang, M. Y. Lee, M. G. Hogg, J. S. Dordick and S. T. Sharfstein, ACS Nano, 2010, 4, 47334743.

L. Conti and E. Cattaneo, Nat. Rev. Neurosci., 2010, 11, 176-87.

37 M. Iwashita, N. Kataoka, K. Toida, Y. Kosodo, Development, 2014, 141, 3793-8. Mierke, B. Frey, M. Fellner, M. Herrmann and B. Fabry, J. Cell Sci., 2011, 124, 369-383.

43 H. Geckil, F. Xu, X. Zhang, S. Moon and U. Demirci, Nanomedicine, 2010, 5, 469-484.

44 J. Zhu and R. E. Marchant, Expert Rev. Med. Devices, 2011, 8, 607-626.

45 A. I. Teixeira, S. Ilkhanizadeh, J. a Wigenius, J. K. Duckworth, O. Inganäs and O. Hermanson, Biomaterials, 2009, 30, 4567-72. 
47 V. B. Bregar, J. Lojk, V. Suštar, P. Veranič, M. Pavlin, V. Šuštar, V. B. Bregar, J. Lojk, V. Suštar, P. Veranič and M. Pavlin, Int. J. Nanomedicine, 2013, 8, 919-31.

48 J.-L. Hoon, W.-K. Wong and C.-G. Koh, Mol. Cell. Biol., 2012, 32, 4246-57. 